\title{
Ultra Wide Band over fibre transparent architecture for High Bit-rate Home Networks
}

\author{
Anna Pizzinat, Franck Payoux, Benoit Charbonnier, Sylvain Meyer \\ France Telecom Research \& Development, 2 Av. Pierre Marzin 22307 Lannion, France \\ Email: name.surname@orange-ftgroup.com
}

\begin{abstract}
Bandwidth hungry services are developing rapidly in home networking and needs for Gigabit Home Networks will appear shortly, following the introduction of Gigabit optical access networks. In addition, ubiquitous wireless connectivity is required by users to connect multiple multimedia devices inside the home. Some wireless standards, such as Ultra Wide Band (UWB), are able to provide Gbit/s data rate but with a limited coverage. In order to extend this coverage, we propose a multipoint to multipoint (MP2MP) radio over fibre (RoF) architecture based on a NxN optical splitter. The UWB MAC layer is able to control the system and no optical MAC layer is required, so that the optical path becomes a "transparent tunnel". Simulations and experimental investigations demonstrate the technical feasibility of this innovative MP2MP RoF architecture.
\end{abstract}

\section{INTRODUCTION}

Today two phenomena are driving the increase of the bit rate needed in a home network. The first one is the multiplication of connected devices (i.e. computers, media centers, media renderers etc...) and of services available to the end user (i.e. domestic storage area network, video-phony and video conferencing, TVoIP, ToIP, etc...). The second phenomenon is the evolution of fiber to the home. As a consequence, a well connected home will need an internal network working at speeds of $1 \mathrm{Gbit} / \mathrm{s}$ by 2010 [1]. Whereas such a target might not be attained by current wired solutions, the large bandwidth of optical fibers makes them the only solution able to guarantee a long life to the network infrastructure and justify the expense for the installation of a new cable. Moreover, using an optical fiber as a home backbone may be seen as the natural prolongation of the optical access.

Additionally, it has to be noted that users have developed a strong preference for wireless connectivity and will require that future systems evolve to higher data rates while remaining wireless. A solution to this requirement can be found in UWB technology [2], [3]. UWB radio systems operate in the frequency range from 3.1 to $10.6 \mathrm{GHz}$ and offer a wireless connectivity up to $1 \mathrm{Gbit} / \mathrm{s}$, but, as a result, are limited in coverage to a few meters $(<10 \mathrm{~m})$.

In this paper we propose to couple radio UWB systems to an optical fibre backbone as shown in figure 1 so to extend the UWB cell coverage to a few hundred meters, i.e. the typical dimension of an in-building network.

Please use the following format when citing this chapter.

Pizzinat. A., Payoux, I:, Charbonnier, B.. Meyer, S., 2007, in Il:IP International Iederation for Information Processing, Volume 256. Ilome Networking. Al Agha. K., Carcelle, X., Pujolle, G.. (Boston: Springer), pp. 39-50. 
The radio home networks will then become a multicellular network with the additional potential of transparently distributing, in parallel with the UWB signal mentioned above, other conventional baseband data signals (GbE etc..) or other radio signals throughout the house such as mobile signals (UMTS, 3G etc...), or different standards of WiFi (e.g. IEEE802.11n) [4], [5]. The important point is the mutualisation of the infrastructure that radio over fiber (RoF) achieves.

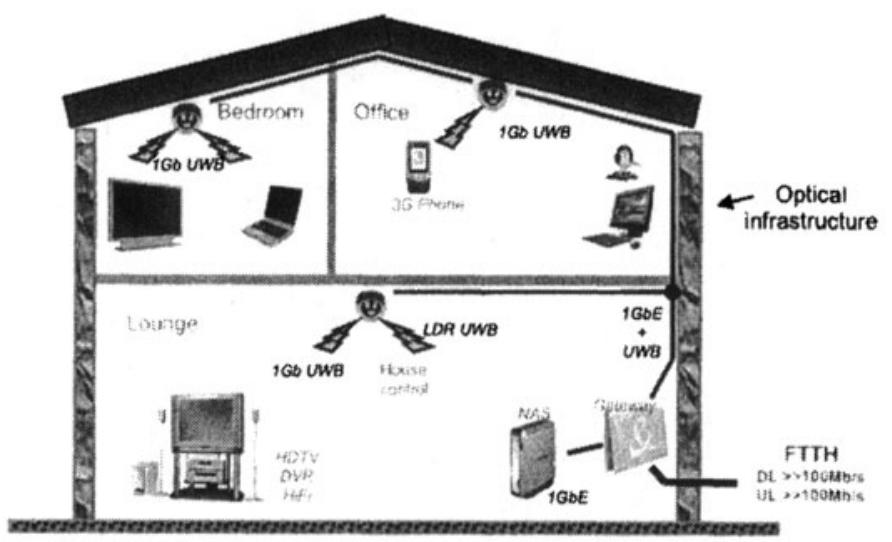

Figure 1: Very High Data Rate Home Area Network supported by an optical infrastructure

In particular, we use the optical fibre as a tunnel that allows enlarging an UWB cell in a completely transparent way, by means of a passive multipoint to multipoint (MP2MP) architecture based on an NxN splitter. This architecture is advantageous because it is equivalent to having all the users in the same room.

RoF has been demonstrated over many types of optical fiber even on legacy multimode fibers [6], but an analysis and experimentation of a complete system keeping into account at the same time for the radio and optical transmission is still lacking. In the following sections we will present the MP2MP architecture and how the RoF system operates. Then, we will introduce the system budget link calculation and compare it with numerical simulations. Finally, we will present the experimental results and come to conclusions.

\section{MP2MP ARCHITECTURE}

\subsection{Physical operation}

The proposed architecture is shown in figure 2 and described in [7]. The key point is the NxN optical splitter thanks to which a signal injected at a 
network input reaches all network outputs. The aim of this optical architecture is to extend the UWB cell coverage, so that it covers a whole apartment or building. Thus, two wireless devices located in two rooms relatively far away from each other can communicate as if they were close to each other. The system operates in the following manner: the signal sent by the first wireless device reaches the RoF transducer. The RoF transducer is mainly composed of one or two antennas, a laser for emission and a photodiode for reception as well as electrical amplifiers to partially compensate for losses induced by propagation in the air. The UWB signal is converted into an electrical signal by the RoF transducer antenna, and this UWB signal directly modulates the laser. The UWB signal in its native format is then carried by the optical physical layer. The signal is transparently propagated through the optical infrastructure. It is in particular broadcasted by the optical splitter to all RoF transducers connected to outputs of the splitter. This can be compared to the diffusion of the radio signal in a room. It has to be noted that the optical splitter is a passive component and does not require any powering. Optical signals are converted into electrical signals by photodiodes in RoF transducers and the original UWB signal is then sent by the antennas in all the other rooms covered by the network. The UWB signal is propagated without any modification at all and it reaches the second wireless device. The entire link is shown in Fig. 3.

As visible in Figure 2, two fibers are used for each transducer, in order to separate propagation directions. One fiber is connected to the laser and the other fiber is connected to the photodiode. The connections of fiber on the optical splitter are done in such a way that every emitted signal is received by all transducers, including itself.

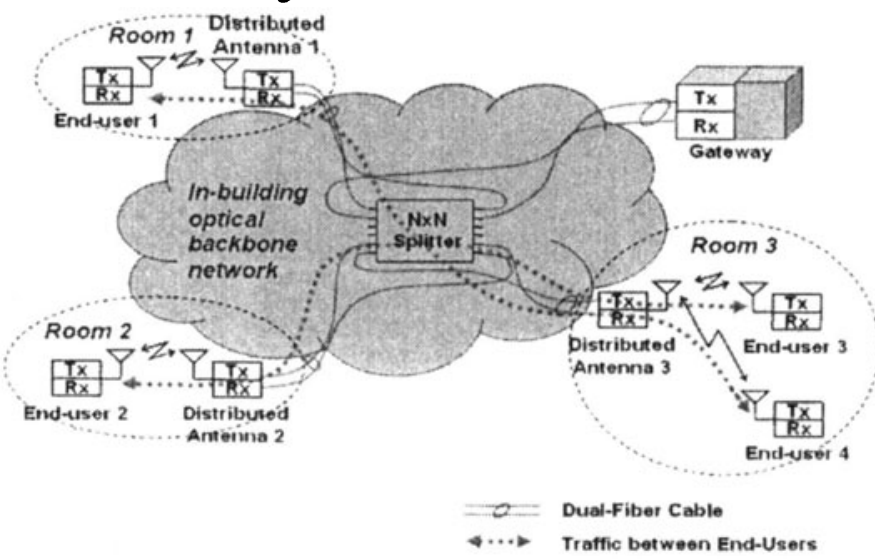

Figure 2: Hybrid Wireless-Optical MP2MP Architecture 


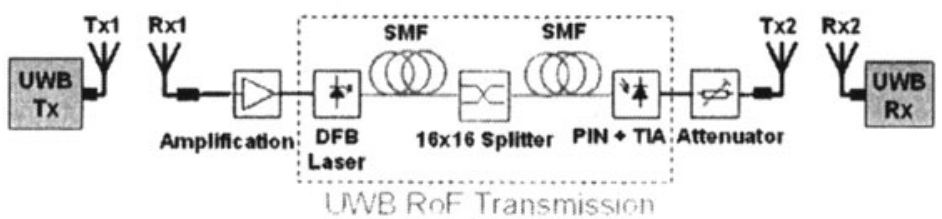

Figure 3: System setup

It has to be noted that the system is independent of the used radio format as far as it respects the laser and photodiode frequency bands. It means that such a system could be used to convey WiFi or $3 \mathrm{G}$ signals to extend the coverage of wireless and mobile networks.

\subsection{MAC layer operation}

With this architecture, the use of different wireless or mobile standards is limited to standards with a MAC layer able to operate with higher reach distances. UWB complies with this requirement and is particularly adequate to this architecture because of the distributed protocol integrated in ECMA 368 MAC layer (DRP: Distributed Reservation Protocol) [3]. There is no Piconet Controller in ECMA 368, each device has the same priority on the network. When a device wants to integrate a network, it first listens to see if grants are available and then it negotiates with all devices of the network to obtain some grants. DRP uses Time Division Multiplexing Access and that enables Quality of Service on the network.

In a classic network solution using two communications technologies (wireless and optical here) there are 2 different MAC layers, in our case, one for the UWB wireless communication, one for the optical communication (Ethernet MAC layer for example). Thanks to radio over fibre technology and to the proposed architecture, the UWB MAC layer alone is sufficient to control communications on the optical and wireless infrastructure. This MAC layer is implemented in end devices and RoF transducers are made of purely physical layer components without any intelligence. This is why the optical infrastructure can be considered as a transparent optical tunnel.

The fact that a device sending a signal receives it because of the connections on the optical splitter is not a problem. This occurs already in a single room with reflections on walls and objects. To avoid the reception of its own signal, the wireless device has an internal switch so that it is either in reception mode or in emission mode but not in both modes in the same time. In addition, in order to eliminate the echo after the end of emission, a guard time is added before switching to reception mode (and vice-versa). In UWB this guard time is $10 \mu \mathrm{s}$, and during this time a signal can propagate over hundreds of meter of fibre and come back to the device without inducing interference problems. So the distance increase is well supported by the UWB MAC layer. 


\subsection{System constraints}

An important choice concerns the fibre type. Indeed, for cost reasons the use of multi-mode fibre and VCSEL may be preferred, but the perennity of such a home backbone has also an important weight. Thus, we study numerically and experimentally the feasibility of the proposed system in the case of single-mode fibres (SMF). We use DFB lasers carefully controlled to operate in linear region and avoid laser clipping. Considering now only the RoF link between two end users, the link to be dimensioned takes the form shown in figure 3.

The power at the output of the transmitting antenna is fixed to -14 $\mathrm{dBm}$ for an OFDM band of $528 \mathrm{MHz}(-41.3 \mathrm{dBm} / \mathrm{MHz}$ is the maximum allowed output power density). The first three UWB bands have been considered.

Electrical amplification is needed before the laser so to partially compensate the free space losses. Optical losses, mainly due to the splitter, are lower than the optical budget given by the laser output power and the PIN photodiode sensitivity. The photoreceiver includes a trans-impedance amplifier that outputs the required electrical power to feed the antenna. The electrical amplification gain and the optical laser output power must be tuned to find a good compromise in terms of link budget and laser linearity, A $16 \times 16$ splitter is considered so to simulate 16 access points.

\section{ANAlytical AND Simulation RESUltS}

The link shown in figure 3 has been evaluated by means of analytical link budget calculations and extensive simulations using Matlab and VPI Transmission Maker.

Fist of all, the system shown in figure 3 has been analytically characterized in terms of link budget. The gain, noise factor and signal to noise ratio (SNR) at the UWB receiver have been calculated for the global link including radio and optical propagation [8]. The received bit error rate (BER) is also estimated from the SNR [9]. For the radio channel only free space losses have been taken into account as a first order approximation. In spite of being valid only for a linear system, the link budget calculation is of fundamental importance for the system dimensioning, i.e.: the ratio of the $\mathrm{NxN}$ coupler and the amplification stage.

For a BER of $10^{-5}$ without coding (that corresponds to error free propagation with coding), we have found that the dimension of the system can reach 16x16 for a propagation distance on each air link up to $10 \mathrm{~m}$.

Besides the system dimensioning, the amplification stage (Figure 3) is also a critical point of the system. This amplification stage is composed of a high gain low noise amplifier (LNA), followed by an electrical variable 
attenuator and a high power amplifier (HPA). The role of the LNA is to compensate the attenuation of the UWB signal due to the propagation in the first air link. The variable attenuator is used to keep constant the RF power at the laser input independently of the propagation distance on the first air link. Finally, the HPA is used to increase the RF power of the UWB signal at the input of the RoF link, in order to compensate the very weak RF gain of the RoF link (about -32.5dB). At the output of the RoF link, an electrical attenuator is used to ensure that the transmitting power of the Tx2 antenna respects the regulation. The parameters for all the system components are shown in Table 1.

\begin{tabular}{|c|c|c|}
\hline \multirow{3}{*}{ DFB Laser } & $\eta_{E O}$ & $0.08 W / A @ I_{\text {Bias }}=80 \mathrm{~mA}$ \\
\hline & $Z_{\text {in }}$ & $50 \Omega$ \\
\hline & $R I N$ & $-140 \mathrm{dBc} / \mathrm{Hz}$ \\
\hline \multirow{3}{*}{$S M F$} & Length & $<500 m$ \\
\hline & Attenuation & $0.2 \mathrm{~dB} / \mathrm{km}$ \\
\hline & $D$ & $16 \mathrm{ps} / \mathrm{hm} / \mathrm{km}$ \\
\hline \multirow{4}{*}{ PIN Photodiode } & $\eta_{O E}$ & $0.95 \mathrm{~A} / \mathrm{W}$ \\
\hline & $Z_{\text {out }}$ & $50 \Omega$ \\
\hline & $Z_{T I A}$ & $500 \Omega$ \\
\hline & $N E P$ & $11.54 \mathrm{pA} / \mathrm{Hz}^{1 / 2}$ \\
\hline Splitter & Ratio & $16 \times 16$ \\
\hline Optical Loss & \multicolumn{2}{|c|}{$\begin{array}{l}15 d B \text { (including } 12 d B \text { optical loss of } 16 \times 16 \\
\text { splitter) }\end{array}$} \\
\hline Antenna & $G_{T \times / R x}$ & $7 d B$ \\
\hline \multirow[b]{2}{*}{$L N A$} & $G$ & $56 d B$ \\
\hline & $N F$ & $0.6 d B$ \\
\hline Var. Atten. & Att. Range & $O d B-30 d B$ \\
\hline \multirow{2}{*}{$H P A$} & $G$ & $35 d B$ \\
\hline & $N F$ & $6 d B$ \\
\hline Rx Pre-Amplifier & $N F$ & $2.5 d B$ \\
\hline
\end{tabular}




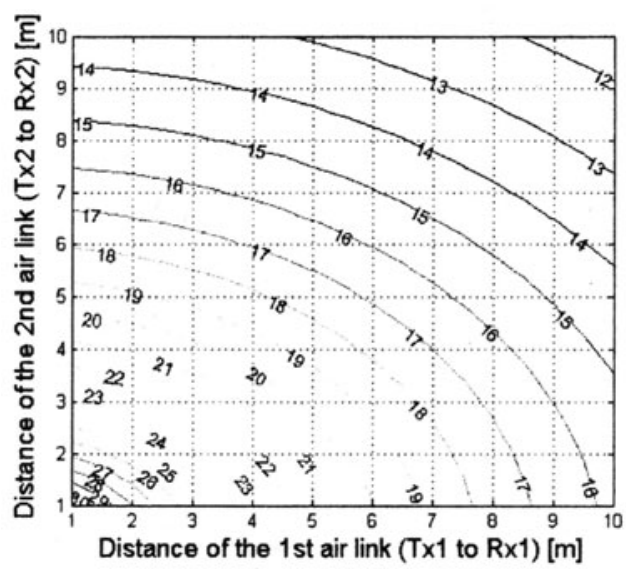

Figure 4 : Estimated SNR (dB) of the first UWB sub-band (3.432GHz) at the system output as a function of the propagation distance on the two air links.

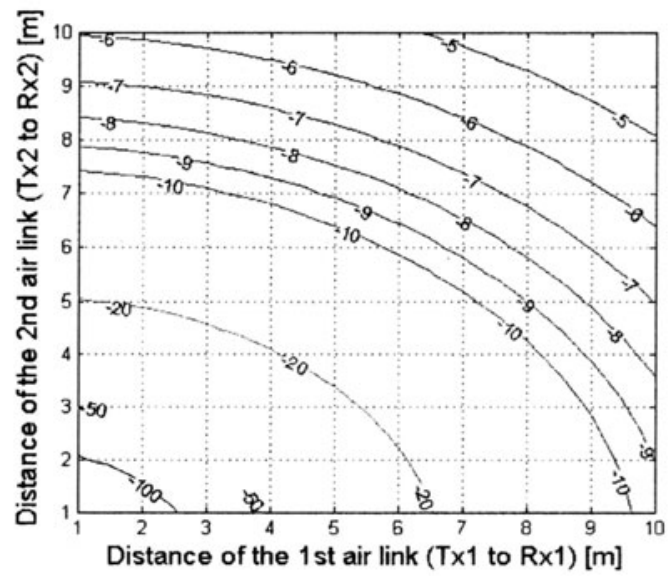

Figure 5: Estimated BER (log10) of the first UWB sub-band as a function of the propagation distance on the two air links.

Two other parameters can also be tuned: the laser input RF power and the laser polarisation current. Their values must be chosen so to avoid laser clipping and third order inter-modulation effects. VPI Transmission Maker simulations and the characterization of a RoF link showed that the RF power injected into the RoF link can be up to $+18 \mathrm{dBm}$ without strong degradation due to the clipping effect of the laser. Therefore, the RF power injected into the RoF link is adjusted to $+15 \mathrm{dBm}$. 
Figures 4 and 5 show the evolutions of the SNR and BER of the UWB signal transmitted through the optical - wireless hybrid system (fig. 3) as a function of the propagation distance on the two air links.

The system behavior has been then numerically simulated in Matlab and VPI in order to analyze also nonlinear effects in the electro-optic conversion. For these simulations, we generate a pseudo random bit sequence at $640 \mathrm{Mbps}$ for each OFDM sub-band, we apply QPSK modulation and obtain the OFDM signal according to [3] but without coding. Afterwards, we extract the UWB baseband signal and load it into VPI. There, the UWB signal is transposed in frequency to create the OFDM sub-band (at $3.432 \mathrm{GHz}$, $3.960 \mathrm{GHz}$ and $4.488 \mathrm{GHz}$ ). Then, the UWB signal is transmitted through the system (described in fig.3) implemented in VPI. The propagation in each air link is simulated by introducing an attenuation that corresponds to free space propagation losses. At the system output, the UWB signal is brought back to base-band and loaded into Matlab for OFDM demodulation and performance evaluation. Figures 6 and 7 show the temporal and spectral behavior of the UWB signal and the QPSK transmitted and received constellations, respectively.
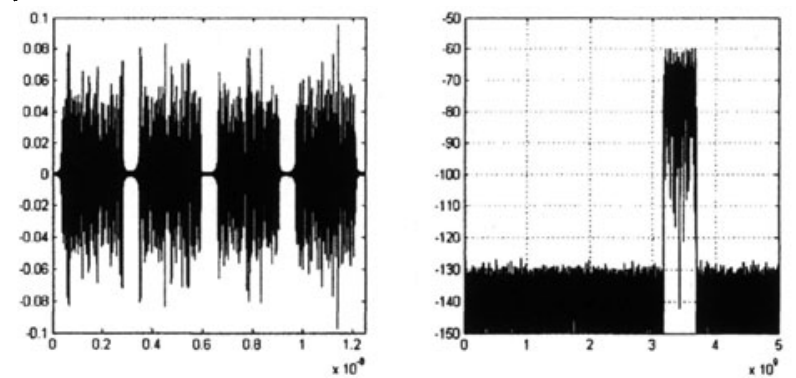

Figure 6: Temporal and spectral representations of the ideal UWB signal (including thermal noise only) in the first OFDM sub-band (3.432GHz).
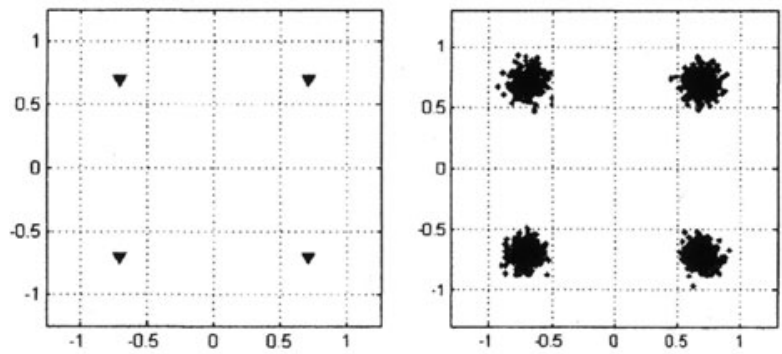

Figure 7: Constellation diagram of DATA sub-carriers: ideal (left) and after transmission (right). 


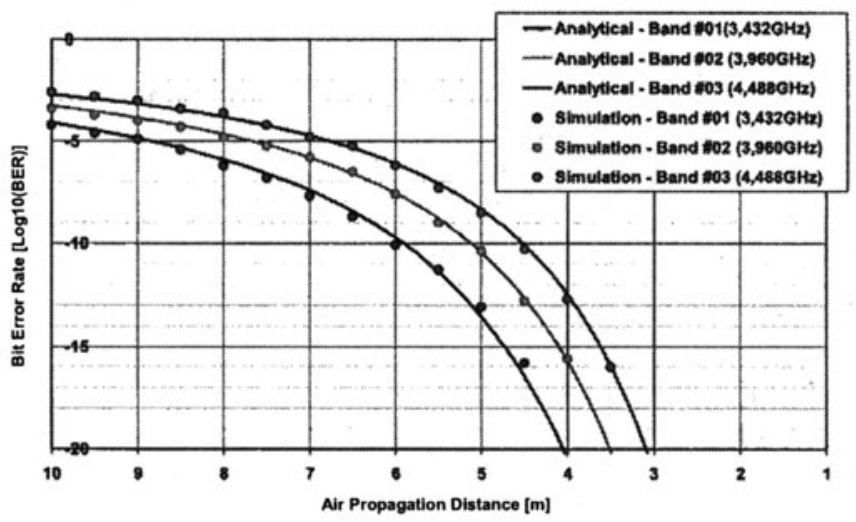

Figure 8: Bit error rate as a function of the transmission distance on the two air links (BER estimated from SNR measurement).

Figure 8 shows the BER as a function of the air propagation distance (supposing equal distances on the two links). A good agreement between link budget calculations and simulation results can be observed.

From this simulation result, we can conclude that the propagation distance on each air link can reach up to $7 \mathrm{~m}$ for a targeted BER of $10^{-5}$.

\section{EXPERIMENTAL RESUlts}

The feasibility of the system shown in fig. 3 has been then experimentally demonstrated. For the moment, we have tested the transmission of the UWB signal on the optical fibre that is named optical tunnel to underline its transparency. We have introduced RF attenuation in order to simulate free space losses of the first air link. Figure 9 shows the system setup and its composition. It has to be noted that this configuration corresponds to the transmission between a user and the gateway in the architecture of figure 2 .

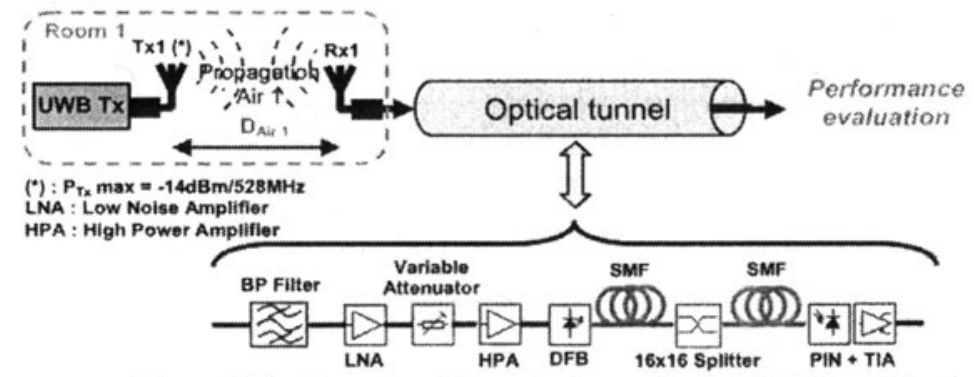

Figure 9: Configuration of the optical tunnel in the MP2MP architecture. 
The principle of the experimental characterization is the same as that applied in the case of the numerical simulation. The UWB signal is loaded into an arbitrary waveform generator (AWG) through a LabVIEW interface (figure 10). After an attenuation stage the signal is sent to the optical tunnel for testing. At the tunnel output, the UWB signal is read by the real time oscilloscope and loaded into the computer. Finally, the UWB signal is demodulated and evaluated by Matlab.

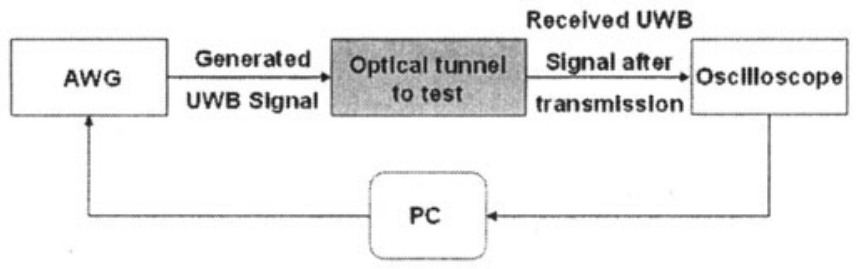

Figure 10: Principle of the experimental test bench (AWG: Arbitrary waveform generator).

The optical tunnel has the parameters given in table 1 . To evaluate the performance of the optical tunnel in the same operating condition as in the hybrid optical - wireless system shown in the figure 3, the RF power of the UWB signal at the tunnel input has to be very weak due to the attenuation of the propagation on the first air link. Therefore, the performance of the tunnel is evaluated for an input $\mathrm{RF}$ power ranging from $-80 \mathrm{dBm}$ to $-50 \mathrm{dBm}$. The BER of the UWB signal transmitted through the tunnel is reported in figure 11, which includes as well the propagation distance on the first air link corresponding to the input RF power. The reported BER is calculated from SNR measurements according to [9] and corresponds to BER before coding.

From this experimental result, we can conclude that the quality of UWB signal at the output of the optical tunnel is always maintained (BER < $10^{-12}$ ) for the propagation distance lower than $10 \mathrm{~m}$ on the first air link. As a consequence, if the system does not include the second air link (communication between the end user and the gateway in fig. 2 for example) the RoF system does not introduce significant penalties on the propagation performance on the air link. 


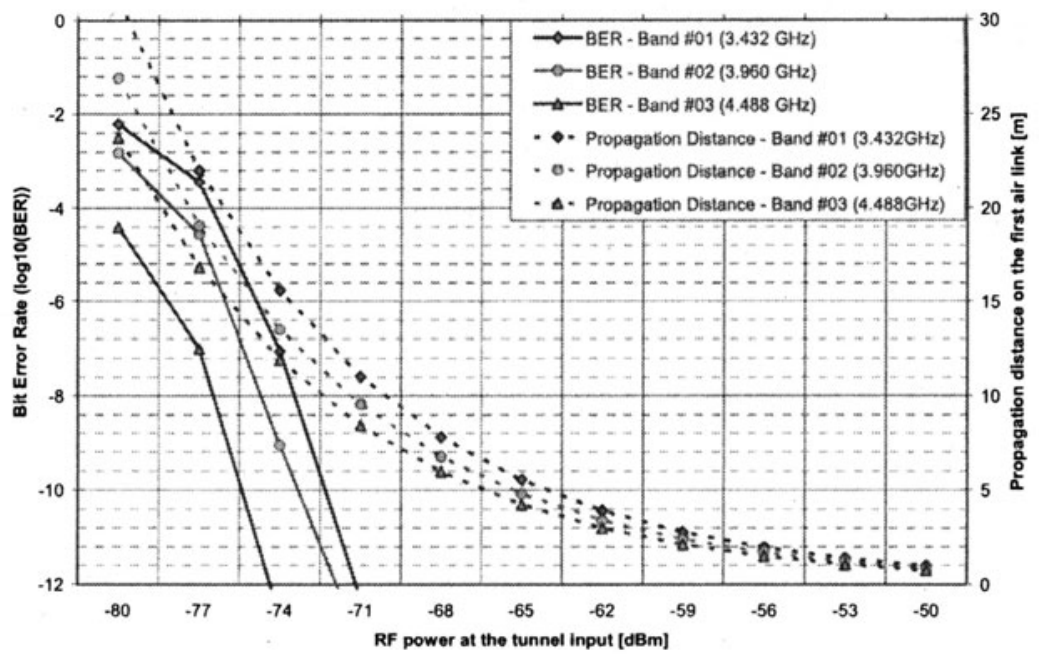

Figure 11: BER of UWB signal transmitted through the optical tunnel as a function of input $R F$ power (BER estimated from SNR measurement).

\section{Conclusions}

We have demonstrated the feasibility of using a hybrid optical - RF wireless system based on single mode fiber to transmit UWB radio signals corresponding to first 3 OFDM sub-bands of $528 \mathrm{MHz}$ in $3.1 \mathrm{GHz}-4.7 \mathrm{GHz}$ frequency range, each carrying $640 \mathrm{Mbps}$. Such results prove the possibility of deploying very high bitrate, UWB based wireless home networks using multipoint-to-multipoint optical transparent architecture.

\section{ACKNOWLEDGEMENTS}

This work has been done in the frame of the national project RNRT/BILBAO and of the ePhotonONE+ VDH Network of Excellence.

\section{REFERENCES}

1. M. Bellec, "Home Broadband Home Area Network", Keynote 3, Tuesday 3rd April, European Wireless 2007

2. FCC, "Rev. Part 15 of the Commission's Rules Regarding UWB Transmission System", ET Docket 98-153, FCC-2-48, 2002.

3. Standard ECMA-368, Geneva, 1st ed. Dec.2005

4. http://www.iece802.org/15/pub/TG3c.html

5. Michael J. Crisp et al, "Demonstration of a Radio over Fibre Distributed Antenna Network for Combined In-building WLAN and 3G Coverage", OFC2007, JThA81 
6. Anna Pizzinat et al, "1.92Gbit/s MB-OFDM Ultra Wide Band Radio Transmission over Low Bandwidth Multimode Fiber", OFC2007, OThM6.

7. P. Guignard et al., "Home network based on CWDM broadcast and select technology", accepted for the presentation at ECOC 2007, Berlin.

8. C. Carlsson et al, "RF transmission over Multimode Fibers using VCSELs-comparing standard and highbandwidth multimode fibers", IEEE J. Lightwave Technol., pp. 1694-1700, 2004.

9. V. J. Urick et al., "Wide-band QAM-over-fiber using phase modulation and interferometric demodulation", IEEE Photonics Technol. Letters, vol. 16, pp. 2374-2376, 2004. 